\title{
Modification of morphine analgesia by venlafaxine in diabetic neuropathic pain model
}

\author{
Krystyna Cegielska-Perun, Magdalena Bujalska-Zadrożny, \\ Helena E. Makulska-Nowak
}

Department of Pharmacodynamics, Medical University of Warsaw, Krakowskie Przedmieście 26/28, PL 00-927 P.O. Box 3, Warszawa 64, Poland

Correspondence: Krystyna Cegielska-Perun, e-mail: kcegielska@wum.edu.pl

\begin{abstract}
:
Background: The purpose of this study was to investigate the influence of single or chronic (21 days) administration of the serotonin and noradrenaline reuptake inhibitor, venlafaxine, on the antinociceptive action of the opioid receptor agonist, morphine, in streptozotocin (STZ)-induced hyperalgesia.

Methods: The studies were performed on male Wistar rats. Changes in nociceptive thresholds were determined using mechanical stimuli. Diabetes was induced by a single administration of STZ $(40 \mathrm{mg} / \mathrm{kg}, \mathrm{im})$.

Results: Venlafaxine was shown to modulate analgesic activity of morphine in STZ-induced hyperalgesia. However, whereas acute co-administration of venlafaxine increased the analgesic activity of morphine, chronic treatment with venlafaxine attenuated opioid efficacy.

Conclusion: Depending on the mode of administration (single or long-term), venlafaxine modulates analgesic activity of morphine. Further investigations are necessary to clarify the mechanisms of these interactions, which may be clinically relevant.
\end{abstract}

Key words:

neuropathic pain, morphine, venlafaxine, rats

Abbreviation: CCI - chronic constriction injury, STZ - streptozotocin

\section{Introduction}

Neuropathies complicating chronic diabetes are accompanied by neuropathic pain. The pathomechanism of painful diabetic neuropathy is complex. Researchers believe that the mechanism of diabetic neuropathy involves metabolic and vascular factors [23].

Diabetic pain management is one of the most difficult challenges in modern medicine. Classical analge- sics, e.g., opioid receptor agonists, exhibit low activity, therefore, other agents, e.g., antidepressants, anticonvulsants are used $[2,19,23]$. Venlafaxine, a selective serotonin and noradrenaline reuptake inhibitor, is a novel antidepressant drug. Venlafaxine has demonstrated antinociceptive action in different animal models of neuropathic pain (CCI, vincristine and STZ) [13-15]. Moreover, venlafaxine has been reported to alleviate pain in patients with diabetic neuropathy, painful polyneuropathy and fibromyalgia [29].

It is commonly believed that antidepressants augment opioid analgesia. There have been many conflicting reports regarding the effect of combined prolonged administration of antidepressants with opioids. 
Some authors demonstrated increased analgesic effects of opioids (morphine) after prolonged therapy with antidepressants (imipramine, clomipramine) [8, 21], whereas others showed decreased analgesic effects of opioids (morphine, fentanyl) after their prolonged administration with antidepressants (amitriptyline, imipramine, fluoxetine, moclobemide, reboxetine) [7, 11].

The purpose of this study was to investigate the effect of single and repeated administrations of venlafaxine on the analgesic action of morphine in the STZ-induced neuropathy pain model, the most commonly used animal model of diabetic neuropathy. Acute injection of STZ causes irreversible damage to some pancreatic B cells, which leads to development of insulin-dependent diabetes. This model results in prolonged mechanical hyperalgesia in rats [1].

\section{Materials and Methods}

\section{Laboratory animals}

This study was conducted according to the guidelines of the Ethical Committee for Experiments on Small Animals, Medical University of Warsaw, which approved the experimental protocols. Male Wistar rats (250-350 g) were housed in a room maintained at the temperature of $20 \pm 2^{\circ} \mathrm{C}$ and under $12-12 \mathrm{~h}$ light-dark cycles. Experimental groups consisted of six rats each. The animals had a free access to food and water, except for a 16-h period before the first experimental session (STZ administration) in the diabetic neuropathy model. Individual animals were used in only one experiment.

\section{Chemicals}

Venlafaxine was obtained from Pliva Hrvatska, Croatia; morphine was obtained from Polfa Warszawa, Poland; STZ [N-(methylnitrosocarbamoyl)- $\alpha$-D-glucosamine] was purchased from Sigma Chemical Co., USA.

\section{Equipment}

Equipment included an analgesimeter (Ugo-Basile, Comerio, Italy), Electronic von Frey anesthesiometer (Stoelting Co., Wood Dale, USA) that progressively exerted stimuli of increasing pressure, and a glucometer (Accu-Check Active, Roche Diagnostics Corp.).

\section{Animal models of neuropathic pain}

STZ-induced (diabetic) painful neuropathy was induced by intramuscular ( $\mathrm{im}$ ) administration of STZ at a dose of $40 \mathrm{mg} / \mathrm{kg}$ of body weight, as described by Nakhoda and Wong [16]. STZ was dissolved in citrate buffer at $\mathrm{pH} 4.5$ and administered in a single dose on the first day of the study into the thigh muscles of the rat leg. Prior to induction of diabetes, the animals fasted for a 16 -h period. Following injection, food and water were available ad libitum during all remaining days of the experiment. Control rats received an equal volume of buffer. Starting on day 3 (72 h after STZ administration), glucose levels were determined using a glucometer. Blood samples were drawn from the tail vein. Permanent hyperglycemia was detected ( $\geq 400 \mathrm{mg} / \mathrm{dl}$ ) in all STZ-treated rats. In vehicletreated animals, glucose levels reached about $90 \mathrm{mg} / \mathrm{dl}$ and remained stable during the entire observation period. STZ-induced hyperglycemia was accompanied by a gradual decrease in body weight, an increase in food consumption, and a considerable increase in water intake.

\section{Drug administration}

STZ was administered as described above.

\section{Preparation of drugs}

Morphine was dissolved in $0.9 \% \mathrm{NaCl}$, whereas venlafaxine was suspended in a $0.5 \%$ water solution of methylcellulose immediately prior to administration.

\section{Administration of drugs}

The venlafaxine dose was selected based on a screening test (data not shown) and the morphine dose - as previously described [4]. Venlafaxine was administered orally $(p o)$ at 10 and $50 \mathrm{mg} / \mathrm{kg}$ doses, whereas morphine was administered subcutaneously $(s c)$ at a $5 \mathrm{mg} / \mathrm{kg}$ dose.

\section{Time schedule}

The antinociceptive action of morphine was determined after a premedication with a single antidepressant dose and after a 21-day antidepressant premedication in the diabetic (STZ)-induced neuropathy model. 


\section{Acute studies}

The influence of single administration of venlafaxine on the activity of morphine was investigated. Venlafaxine and morphine were administered simultaneously on day 19 of the experiment following an STZ injection. At that point, rats had developed hyperalgesia and we observed a similar reduction in their nociceptive thresholds in comparison to the values obtained before neuropathy. The control group received simultaneously morphine and a $0.5 \%$ water solution of methylcellulose. The nociceptive thresholds were determined $30,60,90,120$, 150 , and $180 \mathrm{~min}$ after the morphine injection.

\section{Chronic studies}

The effect of repeated administration of venlafaxine on the analgesic action of morphine was studied. Venlafaxine was administered daily from day 19 to day 39 of the experiment (21 days). This type of repeated treatment closer reflects the use of antidepressants in clinical practice and this model of drug administrations was used in previous publications [3, 7, 11]. On day 39 , measurements of the nociceptive thresholds were conducted in the period from $30 \mathrm{~min}$ to $180 \mathrm{~min}$ after the morphine injection.

At the same time, the control group received a $0.5 \%$ water solution of methylcellulose once daily for 21 days and, on the last day of the experiment, simultaneously received morphine and a $0.5 \%$ water solution of methylcellulose.

\section{Measurement of the nociceptive threshold}

\section{The Randall-Selitto test}

Changes in nociceptive thresholds were determined using mechanical stimuli, which is a modification of the classic paw withdrawal test described by Randall and Selitto [6]. Mechanical stimulation involved the application of progressively increasing pressure to the dorsal surface of the rat's left hind paw using an analgesimeter. The instrument increased the force on the rat's paw at a rate of $32 \mathrm{~g} / \mathrm{s}$. The nociceptive threshold was defined as the force in grams at which the rat attempted to withdraw its paw. Threshold pressure values were recorded. Three threshold measurements were performed daily per rat. The nociceptive threshold values were obtained in triplicate and the mean was derived for further calculations in the statistical analysis.

\section{The von Frey test}

Changes in nociceptive thresholds were evaluated using the von Frey device. Rats were placed in individual plastic boxes with a metal mesh floor and allowed to acclimate for $5 \mathrm{~min}$. The nociceptive testing was done by inserting filaments of gradually increasing thickness through the mesh floor, perpendicularly to the plantar surface of the hind paw. The intensity of mechanical stimuli was increased from 0.1 to $65 \mathrm{~g}$ until the hind paw was withdrawn [10]. Threshold pressure values were recorded. Three threshold measurements were performed daily per rat. Nociceptive threshold values were obtained in triplicate and the mean was derived from further statistical calculations.

\section{Statistical analysis}

Changes in pain threshold were calculated as a percentage of the baseline value according to the following formula:

$$
\% \text { of analgesia }=\left(\frac{B}{A} \times 100 \%\right)-100 \%
$$

where A indicates pressure (in g) at baseline (on study day 1, before STZ administration) and B indicates pressure (in $\mathrm{g}$ ) recorded in the consecutive measurements that were performed after drug administration. Percentage analgesia values calculated for individual animals were used to calculate the means for individual experimental groups and for the statistical analysis.

Results were expressed as the mean \pm standard error of the mean (SEM). The statistical significance of differences between the groups was evaluated by two-way analysis of variance (ANOVA) with replicate measurements, followed by the Fisher post-hoc test; $p$ values of $<0.05$ and $<0.01$ were considered statistically significant. All statistical calculations were performed using Statistica, version 9.

\section{Results}

\section{Effects of STZ on the pain threshold to mechanical stimulation}

Acute injection of STZ $(40 \mathrm{mg} / \mathrm{kg}$, im) gradually decreased the nociceptive threshold, which peaked on day 17 in the modified Randall and Selitto test and on day 16 in the electronic von Frey test, and remained at a similar level on day 39 (Fig. 1A, B) 

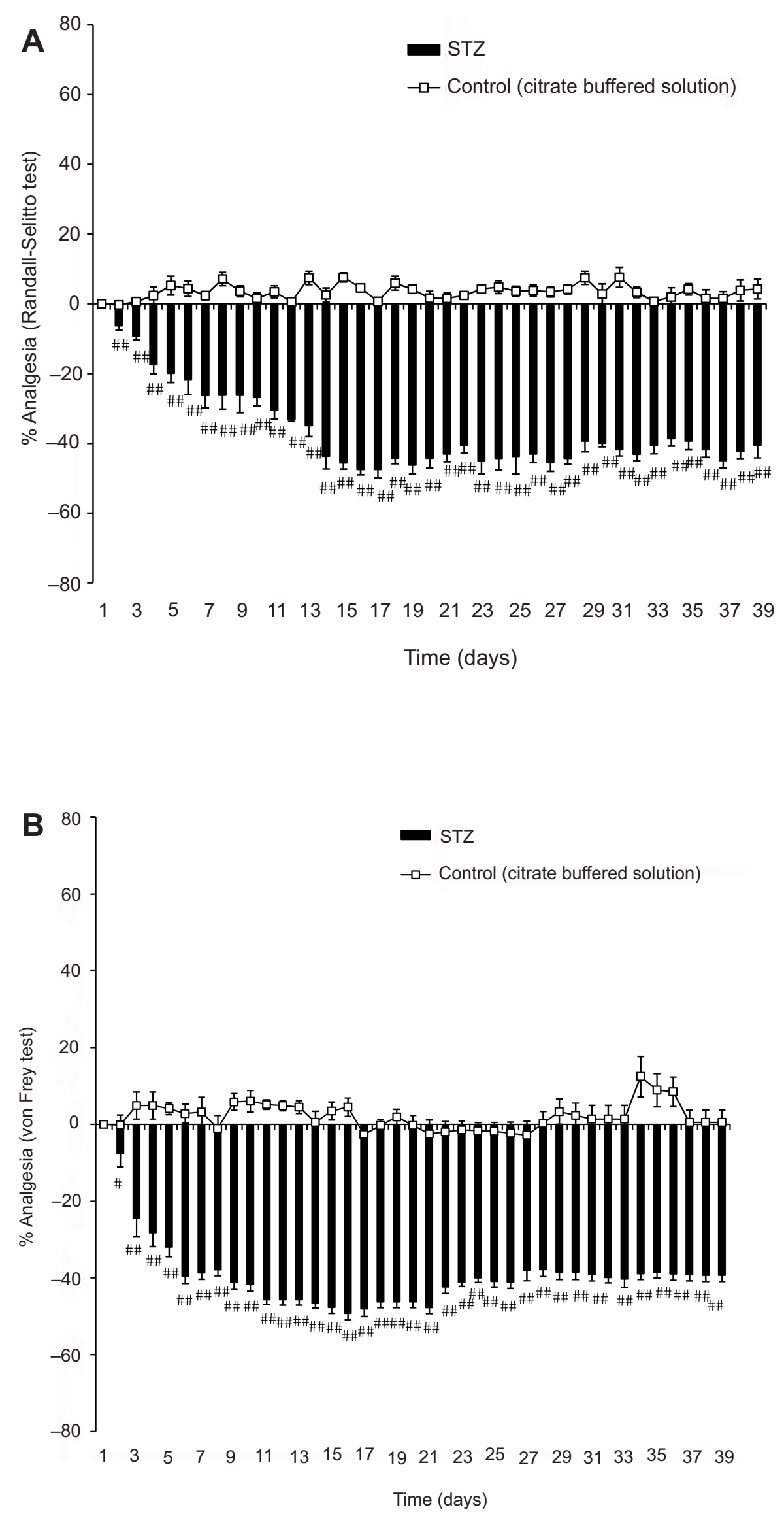

Fig 1. (A) Randall-Selitto test, (B) von Frey test. Influence of streptozotocin (STZ) at dose of $40 \mathrm{mg} / \mathrm{kg}$ im on the pain threshold to mechanical stimuli (study days 1-39). Values are shown as the mean \pm SEM. Control, diabetic rats: $n=6$. STZ vs. control \# $p<0.05$, \#\# $p<0.01$, two-way ANOVA followed by Fisher's test

Time (days) 


\section{Effects of venlafaxine on development of hyperalgesia induced by STZ administration}

A single administration of venlafaxine at a dose of $10 \mathrm{mg} / \mathrm{kg}$, po attenuated STZ-induced hyperalgesia, with the maximal effect observed $30 \mathrm{~min}$ after administration. A higher dose of venlafaxine $(50 \mathrm{mg} /$ $\mathrm{kg}, p o$ ) not only increased the nociceptive threshold but resulted in analgesia observed at 30 and $60 \mathrm{~min}$ after administration (data not shown). We decided to choose the dose $10 \mathrm{mg} / \mathrm{kg}$ of venlafaxine for further studies, because we predicted an increase in the level of pain threshold after repeated administration.

\section{Influence of a single administration of venlafaxine on the activity of morphine in STZ-induced neu- ropathic pain model}

The single administration of morphine $(5 \mathrm{mg} / \mathrm{kg}, s c)$ significantly increased the nociceptive threshold, with the maximal effect at $30 \mathrm{~min}$ of measurement in the STZ-induced model of neuropathic pain. Premedication with venlafaxine $(10 \mathrm{mg} / \mathrm{kg}, p o)$ before morphine administration resulted in an increased antinociceptive activity of morphine, with the maximal analgesic effect observed at $30 \mathrm{~min}$ (Fig. 2A, B).
Fig 2. (A) Randall-Selitto test, (B) von Frey test. The antinociceptive effect of co-administration of venlafaxine (VFX) at dose of $10 \mathrm{mg} / \mathrm{kg}$, po with morphine (MRF) at dose of $5 \mathrm{mg} / \mathrm{kg}, \quad s c$ in a streptozotocin (STZ) model of neuropathy in rats. Values are shown as the mean \pm SEM. Control, diabetic rats: $n=6 . V F X+M R F$ vs. MRF * $p<0.05$ ${ }^{* *} p<0.01 ;$ STZ vs. control, \#\# $\mathrm{p}<0.01$ two-way ANOVA followed by Fisher's test

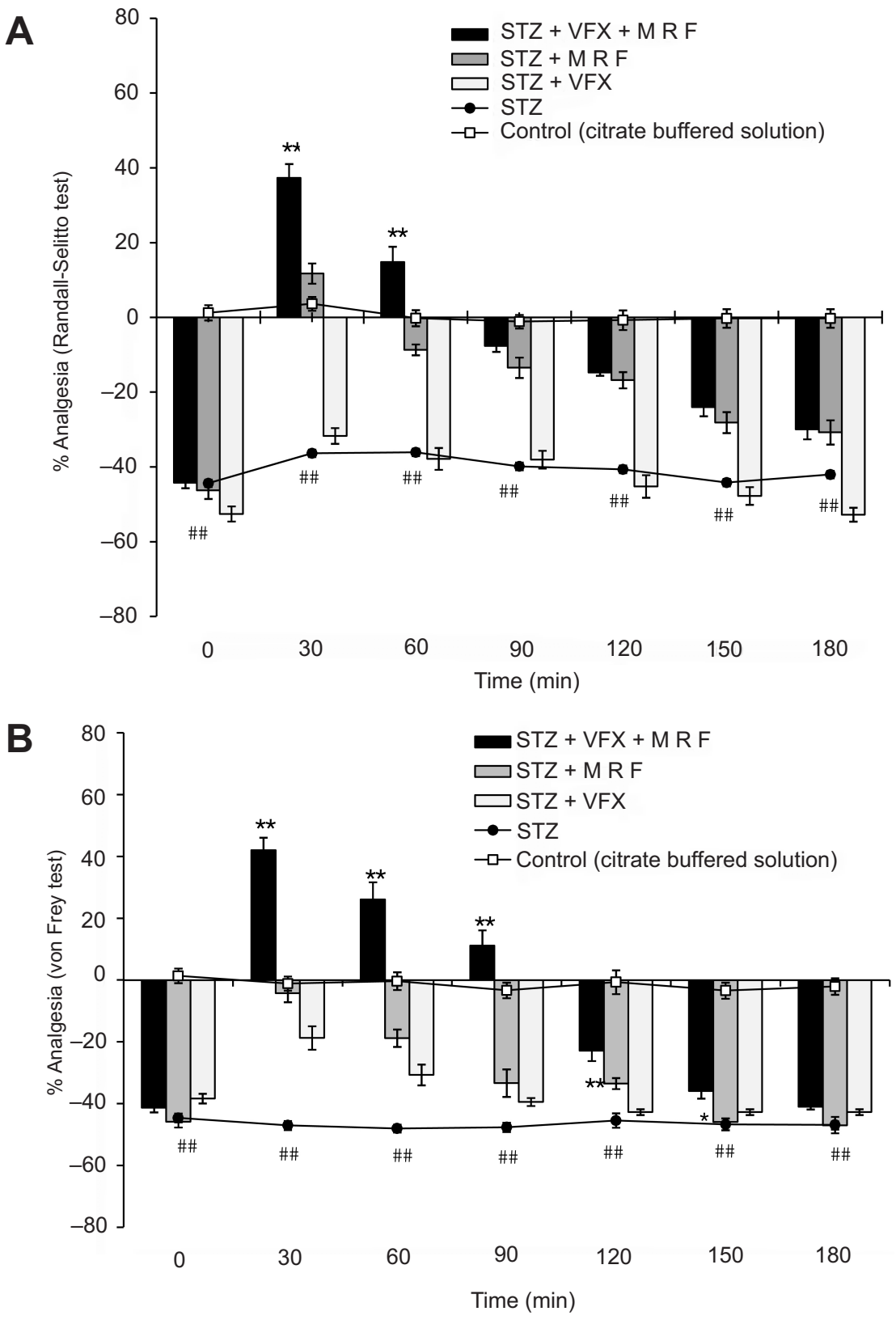


Effects of 21-day premedication with venlafaxine on the activity of morphine in STZ-induced neuropathic pain model

Venlafaxine $(10 \mathrm{mg} / \mathrm{kg}, p o)$ administered for 21 days prior to morphine $(5 \mathrm{mg} / \mathrm{kg}, s c)$ significantly decreased the analgesic action of morphine in diabetic rats (Fig. 3A, B).

\section{Discussion}

Acute injection of STZ (40 mg/kg, im) was shown to gradually decrease the nociceptive threshold, with peak values observed on day 17 in the modified Randall and Selitto test and on day 16 in the electronic von Frey test and maintained at a similar level on day 39.
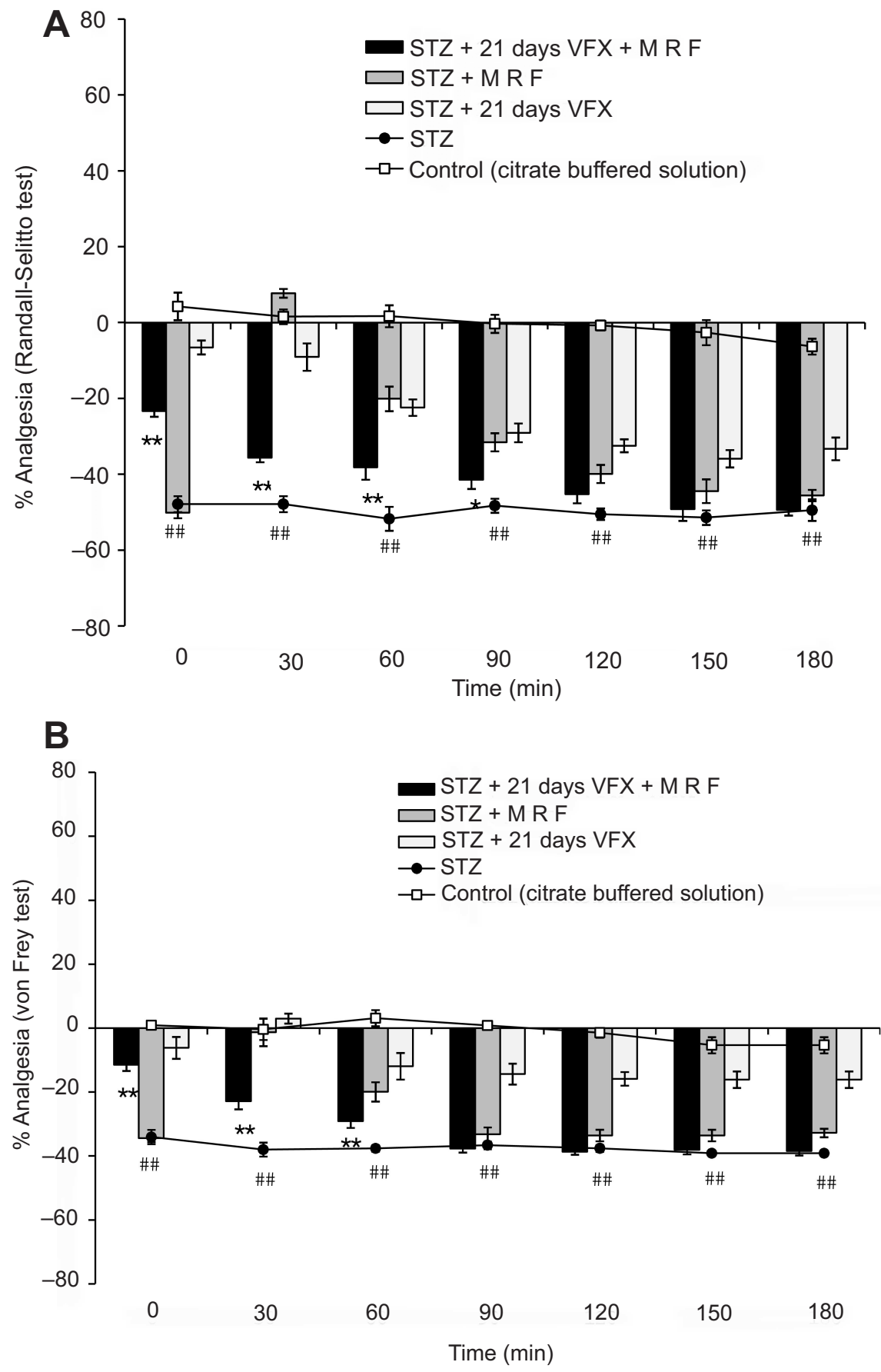

Fig. 3. (A) Randall-Selitto test, (B) von Frey test. The effect of chronic (21 days) administration of venlafaxine (VFX) at dose of $10 \mathrm{mg} / \mathrm{kg}$, po on the antinociceptive activity of morphine (MRF) at dose of $5 \mathrm{mg} / \mathrm{kg}, s c$ in a streptozotocin (STZ) model of neuropathy in rats. Values are shown as the mean \pm SEM. Control, diabetic rats: $n=6.21$ days VFX + MRF vs. MRF ${ }^{*} \mathrm{p}<0.05$, ${ }^{* *} \mathrm{p}<0.01$; STZ vs. control, \#\# $p<0.01$, two-way ANOVA followed by Fisher's test 
We observed development of mechanical hyperalgesia in electronic von Frey test in STZ-induced model of neuropathic pain; however, the animals did not develop tactile allodynia. Mechanical hyperalgesia, observed after three weeks of diabetes, showed a reduced sensitivity to morphine-induced antinociception. This is consistent with numerous earlier reports of a significant reduction in the antinociceptive potency of morphine that was associated with hyperglycemic state in diabetes $[4,5,24]$. Alteration in the $\mu$-opioid receptor function, an increase in the levels of interleukin-1 $\beta$, and accumulation of morphine-3glucuronide were reported in diabetic rats [9].

Repeated (21 days) administration of venlafaxine $(10 \mathrm{mg} / \mathrm{kg}, p o)$ was reported to suppress mechanical hyperalgesia in STZ-induced diabetic rats, whereas a single administration of the drug reduced it only slightly. This result is similar to the findings of another study [13] where a single injection of venlafaxine $(10 \mathrm{mg} / \mathrm{kg}, s c)$ was shown to have no antihyperalgesic effect, while five consecutive injections repeated every hour decreased nociception in the diabetic neuropathic pain model. Serotoninergic and noradrenergic, but not opioidergic, systems are involved in the antihyperalgesic effect of prolonged treatment with venlafaxine $(5 \times 10 \mathrm{mg} / \mathrm{kg}, s c)$ in CCI and STZ-induced models [13, 14].

In the present study, pretreatment with venlafaxine markedly enhanced the analgesic activity of morphine, but prolonged (21 days) administration of venlafaxine completely suppressed the analgesic action of morphine in the STZ-induced neuropathic pain model. Our results are consistent with a previous finding where the selective serotonin reuptake inhibitor (SSRI), fluoxetine, enhanced antinociceptive and antiallodynic effects of morphine in diabetic and sciatic-nerve-ligated mice [24]. Other reports also revealed that acute co-administration of serotonin and noradrenaline reuptake inhibitors (milnacipram and venlafaxine) and a tricyclic antidepressant (doxepin) with tramadol potentiated the antihyperalgesic effect of tramadol in the CCI model of neuropathic pain [17, $26,28]$.

This finding is consistent with other studies in an animal model of acute pain. Juś et al. [11] have reported that a single oral administration of amitriptyline, moclobemide and reboxetine statistically significantly increased the antinociceptive action of fentanyl, whereas 21-day premedication with all investigated antidepressants (amitriptyline, fluoxet- ine, moclobemide, and reboxetine) markedly decreased the analgesic effect of fentanyl in the paw withdrawal test. Another report has also revealed that a single dose of the SSRI (sertraline) increased the antinociceptive effect of morphine, but 14 days of sertraline administration decreased morphine analgesia in mice [18]. Similar results were obtained by Kellstein et al. [12], who also noted that acute treatment with tricyclic antidepressants (clomipramine and desipramine) enhanced analgesia induced by intrathecally-administrated morphine, whereas chronic (21 days) administration of both antidepressants resulted in the loss of enhanced effect of morphine on study day 21 .

The phenomena involved in interaction of these drugs are difficult to explain. However, several studies indicated that a pharmacodynamic, rather than pharmacokinetic, factors are at play. Wattiez et al. [27] provided evidence for opioidergic involvement in the antinociceptive mechanism of antidepressants (clomipramine, milnacipram) after repeated injections in two animal models of neuropathic pain (STZinduced diabetes and CCI). However, Marchand et al. [13] demonstrated that repeated administration (5 injections) of venlafaxine $(10 \mathrm{mg} / \mathrm{kg}, s c)$ suppressed diabetes-induced mechanical hyperalgesia in an opioid-independent manner. Contradictory results concerning the effects of chronic antidepressant treatment on opioid receptor density were also reported. Reisine and Soubrie [20] described a decreased number of opioid receptors in the cortex of rats treated chronically (21 days) with desipramine, however, no change was found in the striatum or hippocampus. Similarly, Stengaard-Pedersen and Schou [25] observed no changes in the cerebral cortex, the basal ganglia or the hippocampus of the forebrain following chronic desipramine treatment (21 days). The results obtained in the present study showed that acute and chronic 21-day pretreatment with venlafaxine did not produce significant changes in the number of $\mu$ opioid receptors in the cerebral cortex in diabetic rats (data not shown). Radioligand binding assays were performed on rat cerebral cortex homogenates, which did not allow for a precise mapping of the brain regions involved in changes of $\mu$ receptor density.

Moreover, a reduced level of endogenous opioids in the brain, down-regulation of adrenergic and serotoninergic receptors, and altered metabolism of opiates may reduce the effectiveness of morphine after chronic premedication with antidepressants [12]. 
STZ-induced diabetes in rats has been extensively used as a model of painful diabetic neuropathy. The animals often display polyuria, diarrhea, and a gradual reduction in body weight. Some authors demonstrated that STZ treatment altered the expression of cytochromes P450 1A2, 2B1 and 4A in rat models of diabetes, which may induce changes in pharmacokinetics of experimental substances [9]. Diabetic rats exhibit many complications seen in patients with diabetic neuropathy, including hyperalgesia and progressive loss of sensory nerve conduction, impaired intraneuronal blood flow as well as micro- and macrovascular reactivity. However, short-term insulindeficient diabetes, does not result in segmental demyelination and fiber degeneration found in patients [22].

\section{Acknowledgments:}

We wish to thank Pliva Hrvatska, Croatia, for the gifts of venlafaxine and Polfa Warszawa, Poland, for the gifts of morphine. We thank Robert Wrzesień for his help in decapitation of animals.

\section{References:}

1. Aley KO, Levine JD: Different peripheral mechanisms mediate enhanced nociception in metabolic/toxic and traumatic painful peripheral neuropathies in the rat. Neuroscience, 2002, 111, 389-397.

2. Attal N, Cruccu G, Baron R, Haanpää M, Hansson P, Jensen TS, Nurmikko T: European Federation of Neurological Societies: EFNS guidelines on the pharmacological treatment of neuropathic pain: 2010 revision. Eur J Neurol, 2010, 17, 1113-1188.

3. Benbouzid M, Choucair-Jaafar N, Yalcin I, Waltisperger E, Muller A, Freund-Mercier MJ, Barrot M: Chronic, but not acute, tricyclic antidepressant treatment alleviates neuropathic allodynia after sciatic nerve cuffing in mice. Eur J Pain, 2008, 12, 1008-1017.

4. Bujalska M, Malinowska E, Makulska-Nowak H, Gumułka SW: Magnesium ions and opioid agonist activity in streptozotocin-induced hyperalgesia. Pharmacology, 2008, 82, 180-186.

5. Courteix C, Bardin M, Chantelauze C, Lavarenne J, Eschalier A: Study of the sensitivity of the diabetesinduced pain model in rats to a range of analgesics. Pain, 1994, 57, 153-160.

6. Courteix C, Eschalier A, Lavarenne J: Streptozocininduced diabetic rats: behavioural evidence for a model of chronic pain. Pain, 1993, 53, 81-88.

7. Godefroy F, Butler SH, Weil-Fugazza J, Besson JM: Do acute or chronic tricyclic antidepressants modify morphine antinociception in arthritic rats? Pain, 1986, $25,233-244$.
8. Gutiérrez M, Ortega-Alvaro A, Gibert-Rahola J, Micó JA: Interactions of acute morphine with chronic imipramine and fluvoxamine treatment on the antinociceptive effect in arthritic rats. Neurosci Lett, 2003, 352, 37-40.

9. Hasegawa Y, Kishimoto S, Shibatani N, Nomura H, Ishii $\mathrm{Y}$, Onishi M, Inotsume $\mathrm{N}$ et al.: The pharmacokinetics of morphine and its glucuronide conjugate in a rat model of streptozotocin-induced diabetes and the expression of MRP2, MRP3 and UGT2B1 in the liver. J Pharm Pharmacol, 2010, 62, 310-314.

10. Hoybergs YM, Meert TF: The effect of low-dose insulin on mechanical sensitivity and allodynia in type I diabetes neuropathy. Neurosci Lett, 2007, 417, 149-154.

11. Juś A, Bujalska M, Makulska-Nowak HE: Modification of fentanyl analgesia by antidepressants. Pharmacology, 2010, 85, 48-53.

12. Kellstein DE, Malseed RT, Ossipov MH, Goldstein FJ: Effect of chronic treatment with tricyclic antidepressants upon antinociception induced by intrathecal injection of morphine and monoamines. Neuropharmacology, 1988, 27, 1-14.

13. Marchand F, Alloui A, Chapuy E, Hernandez A, Pelissier T, Ardid D, Eschalier A: The antihyperalgesic effect of venlafaxine in diabetic rats does not involve the opioid system. Neurosci Lett, 2003, 342, 105-108.

14. Marchand F, Alloui A, Chapuy E, Jourdan D, Pelissier T, Ardid D, Hernandez A et al.: Evidence of a monoamine mediated, opioid-independent, antihyperalgesic effect of venlafaxine, a non-tricyclic antidepressant, in a neurogenic pain model in rats. Pain, 2003, 103, 229-235.

15. Marchand F, Alloui A, Pelissier T, Hernández A, Authier N, Alvarez P, Eschalier A et al.: Evidence for an antihyperalgesic effect of venlafaxine in vincristine-induced neuropathy in rat. Brain Res, 2003, 980, 117-120.

16. Nakhoda A, Wong HA: The induction of diabetes in rats by intramuscular administration of streptozotocin. Experientia, 1979, 35, 1679-1680.

17. Onal A, Parlar A, Ulker S: Milnacipran attenuates hyperalgesia and potentiates antihyperalgesic effect of tramadol in rats with mononeuropathic pain. Pharmacol Biochem Behav, 2007, 88, 171-178.

18. Pakulska W: Influence of sertraline on the antinociceptive effect of morphine, metamizol and indomethacin in mice. Acta Pol Pharm Drug Res, 2004, 61, 157-163.

19. Przeklasa-Muszyńska A, Dobrogowski J: Transdermal buprenorphine in the treatment of cancer and non-cancer pain - the results of multicenter studies in Poland. Pharmacol Rep, 2011, 63, 935-948.

20. Reisine T, Soubrie P: Loss of rat cerebral cortical opiate receptors following chronic desimipramine treatment. Eur J Pharmacol, 1982, 77, 39-44.

21. Rosland JH, Hunskaar S, Hole K: Modification of the antinociceptive effect of morphine by acute and chronic administration of clomipramine in mice. Pain, 1988, 33, 349-355.

22. Sigaudo-Roussel D, Fromy B, Saumet JL: Diabetic neuropathy in animal models. Drug Discov Today Dis Models, 2007, 4, 39-44.

23. Smith HS, Afgoff CE: Pharmacological treatment of diabetic neuropathic pain. Drugs, 2011, 71, 557-589. 
24. Sounvoravong S, Nakashima MN, Wada M, Nakashima $\mathrm{K}$ : Modification of antiallodynic and antinociceptive effects of morphine by peripheral and central action of fluoxetine in a neuropathic mice model. Acta Biol Hung, 2007, 58, 369-379.

25. Stengaard-Pedersen K, Schou M: Opioid receptors in the brain of the rat following chronic treatment with desipramine and electroconvulsive shock. Neuropharmacology, 1986, 25, 1365-1371

26. Uyar M, Onal A, Uyar M, Dogru A, Soykan N: The antinociceptive effect of tramadol-venlafaxine combination on the paw withdrawal threshold in a rat model of neuropathic pain. Methods Find Exp Clin Pharmacol, 2003, 25, 361-365.

27. Wattiez AS, Libert F, Privat AM, Loiodice S, Fialip J, Eschalier A, Courteix C: Evidence for a differential opioidergic involvement in the analgesic effect of antidepressants: prediction for efficacy in animal models of neuropathic pain? Br J Pharmacol, 2011, 163, 792-803.

28. Wrzosek A, Obara I, Wordliczek J, Przewlocka B: Efficacy of tramadol in combination with doxepin or venlafaxine in inhibition of nociceptive process in the rat model of neuropathic pain: an isobolographic analysis. J Physiol Pharmacol, 2009, 60, 71-78.

29. Yee-Chi L, Phoon-Ping Ch: A review of SSRIs and SNRIs in neuropathic pain. Expert Opin. Pharmacother, 2010, 11, 2813-2815.

Received: November 30, 2011; in the revised form: April 16, 2012; accepted: May 30, 2012. 